\title{
Close correlation between MEK/ERK and Aurora-B signaling pathways in sustaining tumorigenic potential and radioresistance of gynecological cancer cell lines
}

\author{
FRANCESCO MARAMPON $^{1,2^{*}}$, GIOVANNI LUCA GRAVINA ${ }^{1,2 *}$, VALDIMIR M. POPOV ${ }^{3,4}$, \\ LUCA SCARSELLA $^{1}$, CLAUDIO FESTUCCIA ${ }^{2}$, MARIA EMILIA LA VERGHETTA ${ }^{2}$, SILVIA PARENTE ${ }^{2}$, \\ MANUELA CERASANI ${ }^{2}$, GEMMA BRUERA ${ }^{5}$, CORRADO FICORELLA ${ }^{5}$, ENRICO RICEVUTO ${ }^{5}$, \\ VINCENZO TOMBOLINI ${ }^{6}$, ERNESTO DI CESARE ${ }^{1,2}$ and BIANCA MARIA ZANI ${ }^{1}$
}

\footnotetext{
${ }^{1}$ Department of Biotechnological and Applied Clinical Sciences, University of L'Aquila; ${ }^{2}$ Department of Biotechnological and Applied Clinical Sciences, Division of Radiotherapy and Radiobiology Laboratory, San Salvatore Hospital, University of L'Aquila, I-67100 L'Aquila, Italy; ${ }^{3}$ LIPOGEN LLC, Mount Laurel, NJ 08054; ${ }^{4}$ Department of Cancer Biology and Medical Oncology, Kimmel Cancer Center, Thomas Jefferson University, Philadelphia, PA 19107, USA; ${ }^{5}$ Medical Oncology, S. Salvatore Hospital, University of L'Aquila,

L'Aquila, I-67100; ${ }^{6}$ Department of Radiological Sciences-Oncology and Pathological Anatomy, and Department of Radiotherapy, University of Rome 'La Sapienza' I-00161 Rome, Italy
}

Received July 11, 2013; Accepted August 30, 2013

DOI: $10.3892 /$ ijo.2013.2167

\begin{abstract}
Both Aurora-A and -B kinases have been implicated in tumorigenesis; and as such, they represent an attractive therapeutic target. Recent studies found that Aurora-A is a downstream target of mitogen-activated protein kinase 1/ ERK2, while Aurora-B has been found to be a prognostic/ predictive therapeutic target for epithelial cancer. In a wide range of human cancers, the Ras/Raf/MEK/ERK/MAP kinase pathway is enhanced and the cellular response to growth signals is known to increase. The purpose of this study was to investigate whether the MEK/ERK cascade regulates tumorigenic signaling and radioresistance via the AuroraB-mediated pathway in a panel of gynecological cancer cell lines. Exponentially growing human endometrial (Ishikawa), cervical (HeLa), cervical (CASKI) and vulva ( $\mathrm{SiHa}$ ) cancer cells were used in culture treated with either control or MEK/ ERK inhibitor or AZD1152 before and after irradiation. Western blotting, ERK1/2 siRNA transfection, growth assay in modified monolayer, Annexin $\mathrm{V}$ and migration/invasion assays were performed. The specific MEK/ERK inhibitor U0126 decreased the tumorigenic potential and improved the
\end{abstract}

Correspondence to: Dr Francesco Marampon, Department of Biotechnological and Applied Clinical Sciences, Division of Radiotherapy and Radiobiology Laboratory, San Salvatore Hospital, University of L'Aquila, Via Vetoio, I-67100 L'Aquila, Italy

E-mail: f.marampon@gmail.com

"Contributed equally

Key words: U0126, gynecological cancer, Aurora-B, MEK/ERK inhibitor, radiotherapy radiation response in all cellular models. The modulation of radioresponse upon U0126 treatment positively correlated with the inhibition of phospho-ERKs and the reduction of Aurora-B kinase expression. In addition, upon U0126 treatment DNA-PKcs protein expression was found to be downregulated, indicating that the improved radiation response may be caused by decreased DNA double-strand damage repair mechanisms. The knockdown of ERK by siRNA confirmed the MEK/ERK-dependent Aurora-B kinase expression. The use of AZD1152, a selective Aurora-B inhibitor, counteracted tumorigenic potential and radioresistance phenotype by highly increasing apoptotic mechanisms in all gynecological cancer cell lines used. Evidence from our experiments show that tumorigenic potential and radiation response in gynecological cancer cells may ensue from a MEK/ERK or Aurora-B inhibition. Together with the close correlation of MEK/ERK and Aurora-B protein expression, this study underlines the potential role of a MEK/ERK/Aurora-B axis whose interruption recovers the antitumor effects of radiotherapy.

\section{Introduction}

Even though cervical, endometrial and ovarian cancers are relatively common, vulvar, vaginal, and fallopian tube cancers, and choriocarcinomas are very rare (1). Treatment of these malignancies requires a multimodal approach combining surgery, radiotherapy (RT) and/or chemotherapy. Although overall statistics have improved considerably, the outcome for patients with high-risk disease remains relatively poor, which points to a clear need for new therapeutic strategies. In situations like these, radiation therapy can be used to prevent local cancer recurrences after surgery (2-4). It has been clarified that the radiation resistance is associated with the abnormal 
expression of activated oncogenes, including Ras $(5,6)$ and Aurora-B (7). The Ras/Raf/mitogen-activated protein kinase kinase (MEK)/extracellular signal-regulated kinase (ERK) cascade regulates proliferation, differentiation, survival, motility (8-18) and when constitutively activated, mediates resistance to ionizing radiation (15). The aurora kinases are a family of oncogenic serine/threonine kinases involved in the mitotic (M) phase of the cell cycle, acting to establish the mitotic spindle, bipolar spindle formation, alignment of centrosomes on mitotic spindle, centrosome separation, cytokinesis and monitoring of the mitotic checkpoint (19-22). There are three known aurora kinases (Aurora-A, -B and -C) in human neoplastic and non-neoplastic tissues (19,22-26). Inhibition of aurora kinase activity leads to catastrophic errors of mitosis, defective cytokinesis, misaligned centrosomes, mitotic spindle malformation and apoptosis (26-30). However, the role of aurora kinases in radioresistance phenomena remains unclear. The Ras/Raf/MEK/ERKs pathway positively regulates Aurora-B expression at the transcriptional level (31). DNA double-strand break is critical in DNA lesions induced by radiation. In mammalian cells the repair of these lesions occurs by non-homologous end joining (NHEJ) requiring $\mathrm{Ku} 70 / \mathrm{Ku} 86$ and the recruitment of the catalytic subunit of DNA-dependent PK (DNAPKcs). DNAPKcs is implicated in radioresponsiveness of embryonal rhabdomyosarcomauterine-derived cell lines (32-34) and human glioblastoma cell lines $(35,36)$. DNA-PKcs are necessary for genomic stability whereas abnormal levels in cancer cell may contribute to cell proliferation, radioprotection eventually contributing to oncogenic phenotype (37-40). Herein we investigated whether MEK/ERK pathway, collaborating with Aurora-B kinase, sustains the tumorigenic potential and radioprotection in a panel of gynecological cancer cell lines.

\section{Materials and methods}

Cell culture, treatment and radiation exposure. The human Ishikawa endometrial cancer cell line was kindly provided by Professor Marcello Maggiolini (University of Calabria, Italy). The human HeLa cervical cancer cell line was kindly provided by Professor Roberto Maggio (University of L'Aquila, Italy), while the human CASKI cervical- and SiHa vulva-cancer cell line were kindly provided by Professor P.J.F. Snijders (VU Medisch Centrum, Germany). Tumor cell lines were cultured in the appropriate medium supplemented with $10 \%$ fetal calf serum (FCS). Treatments with $10 \mu \mathrm{mol} / 1 \mathrm{MEK} / \mathrm{ERK}$ inhibitor U0126 (1,4-diamino-2, 3-dicyano-1,4-bis[2-aminophenylthio] butadiene; Promega) or with AZD1152 (60 nM), an Aurora-B kinase inhibitor, was done as shown in the figures. Radiation was delivered at room temperature using an X-ray LINAC at the dose rate of $2.5 \mathrm{~Gy} / \mathrm{min}$. For clonogenic survival assay, exponentially growing cells, diluted to appropriate densities, were plated with complete medium in presence of U0126, AZD1152 or vehicle/control [dimethyl sulfoxide (DMSO) $0.1 \%$ ] and then irradiated with graded doses (0-2-4-6 Gy). Cells were then cultured in drug-free medium for 14 days, fixed with methanol/acetic acid (10:1, v/v) and stained with crystal violet. Colonies containing $>50$ cells were counted. The plating efficiency (PE) was calculated as the number of colonies observed/the number of cell plated; the surviving fraction (SF) was calculated as: colonies counted/cells seeded (PE/100).

Cell proliferation assay. Cells from adherent culture were counted using hemocytometer and tested for exclusion of trypan blue. Results represent the average of triplicate experiments, including standard error.

Western blot analysis. Proteins of whole cell lysates were assessed using the Lowry method (40) and equal amounts of proteins were separated on SDS-PAGE. The proteins were transferred to a nitrocellulose membrane (Schleicher \& Schell Bioscience GmbH, Germany) by electroblotting. Immunoblottings were performed with the following antibodies directed against c-Myc, ERK1/2, phospho-ERK1/2, p27, cyclin D1, CDK2, Aurora-B, DNAPKcs and $\alpha$-tubulin (B-7) (all from Santa Cruz Biotechnology, Santa Cruz, CA, USA). Peroxidase-conjugate anti-mouse or anti-rabbit IgG (Amersham-Pharmacia Biotech, UK or Santa Cruz) were used for enhanced chemiluminescence (ECL) detection.

Cell cycle and apoptosis analysis by flow cytometry. After the appropriate treatments, cells $\left(1 \times 10^{6}\right)$ were fixed for $30 \mathrm{~min}$ in $70 \%$ ethanol and pelleted by centrifugation (720 g; $5 \mathrm{~min}$ ). After removal of ethanol, cells were incubated and resuspended in $1 \mathrm{ml}$ of DNA staining solution (PBS containing $200 \mathrm{mg} / \mathrm{ml}$ RNase A, $20 \mathrm{mg} / \mathrm{ml}$ propidium iodide plus $0.1 \%$ Triton $\mathrm{X}-100$ ) and left at room temperature for $60 \mathrm{~min}$. Ten thousand events per sample were acquired using a FACScan flow cytometer (Becton-Dickinson, San Jose, CA, USA) and the percentage of cells in $G_{1} / S, G_{2} / M$ and Sub- $G_{2} / M$ phases of the cell cycle were determined using CellQuest software (BectonDickinson). Apoptosis was analyzed by using Annexin V staining (GenScript, Piscataway, NJ, USA). All cells were then measured on a FACScan flow cytometer with an argon laser at $488 \mathrm{~nm}$ for excitation and analyzed using CellQuest software. Apoptotic cells were detected by the percentage of Annexin V stained cells. The results were expressed as the percentage of death by apoptosis induced by a specific treatment.

Soft agar clonogenic assays. Soft agar assays were performed as previously described (34). Briefly, $2 \times 10^{3}$ cells were suspended in $0.3 \%$ Bacto-agar (Life Technologies) containing the appropriate medium supplemented with $10 \% \mathrm{FCS}$ and/or $10 \mu \mathrm{M}$ U0126 and seeded in 60-mm cell culture plates. The plates were incubated at $37^{\circ} \mathrm{C}$ for 14 days. The numbers of colonies containing $\geq 6$ cells were counted.

Small interfering RNA transfection. Tumor cells $\left(1.5 \times 10^{5}\right.$ cells/ well) were plated in 6-well plates and grown in the appropriate medium supplemented with $10 \%$ FCS for 2 days. These cells were transfected with ERK1siRNA and ERK2 siRNA (sc-29308 and sc-44224 respectively, Santa Cruz Biotechnology). All siRNA duplexes were transfected using Oligofectamine reagent (Invitrogen) according to the manufacturer's instructions. After ERK transfection, cells were grown in the appropriate medium for $72 \mathrm{~h}$.

Invasion and migration assays. Transwell membrane (Corning Costar Corp.) was used. Cancer cells were trypsinized, washed 
A
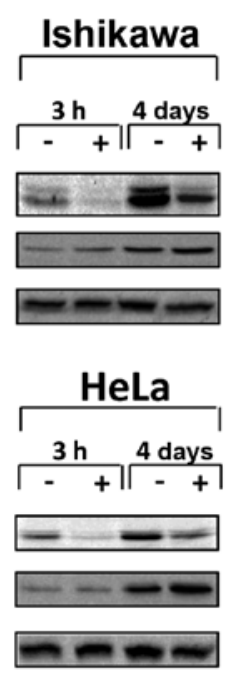

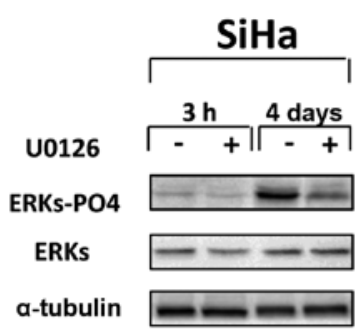

CASKI

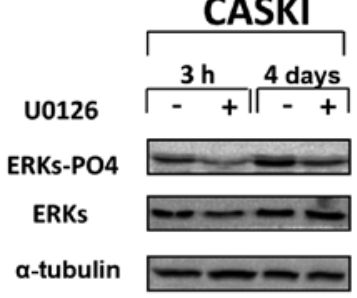

B
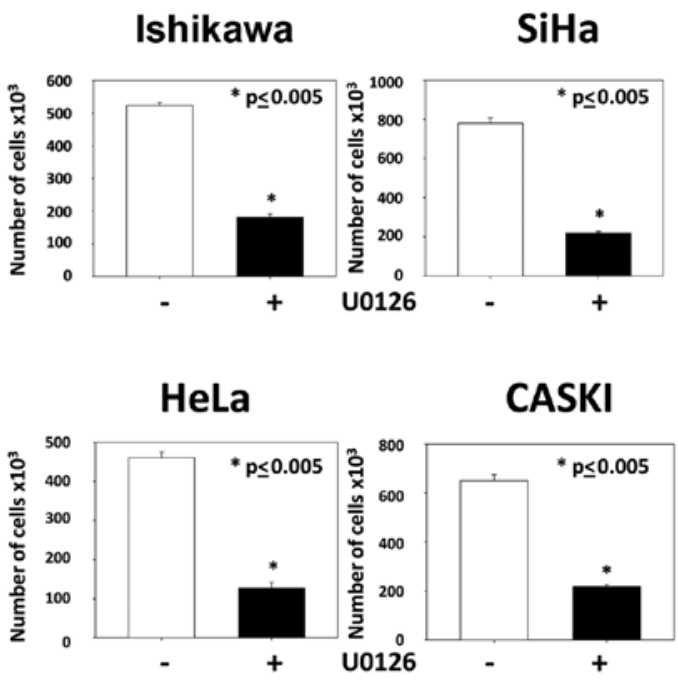

D

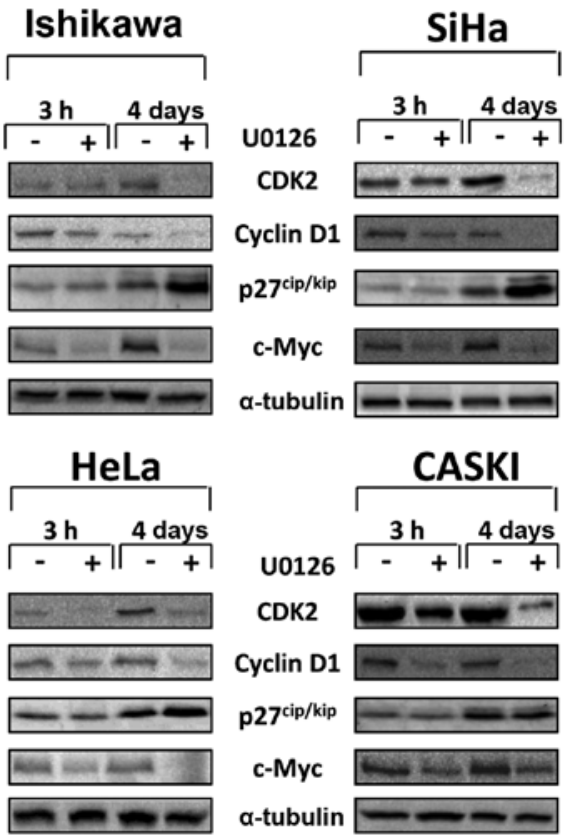

Figure 1. U0126 induces ERK inhibition and cell growth arrest in $\mathrm{G}_{1} / \mathrm{S}$ phase of the cell cycle of Ishikawa, SiHa, HeLa and CASKI gynecological cancer cell lines. (A) Expression of protein kinases ERK1/2-P04 and ERK1/2 in untreated (-) or $3 \mathrm{~h}$ and 4 days U0126-treated (+) Ishikawa, SiHa, HeLa and CASKI cancer cell lines. $\alpha$-tubulin was blotted as loading control. (B) Growth curve of Ishikawa, SiHa, HeLa and CASKI cancer cell lines untreated (-) or U0126-treated (+) for 4 days. Each point of the curve is the average \pm SEM of three samples. (C) Histogram showing the number of Ishikawa, SiHa, HeLa and CASKI cells in $\mathrm{G}_{0} / \mathrm{G}_{1}$ phase in untreated (-) and U0126-treated cancer cells (+) for $24 \mathrm{~h}$. Similar results were obtained in three separate experiments. (D) Cell lysates from Ishikawa, SiHa, HeLa and CASKI cells untreated (-) or treated (+) with U0126 for $3 \mathrm{~h}$, or 4 days were analyzed by immunoblotting with specific antibodies for indicated proteins. $\alpha$-tubulin expression shows the loading of samples. Similar results were obtained in three different experiments.

and kept suspended in the appropriate medium without FCS. To the lower wells of the chambers, migration-inducing medium (with $10 \%$ FCS) was added. Upper wells were filled with serum-free medium with cells $(20,000$ cells per well) in the absence or in the presence of the appropriate treatments. After $8 \mathrm{~h}$, filters were removed and fixed with methanol and subsequently the cells on the upper side were wiped off using a Q-tip. Filters were stained with $20 \%$ Giemsa solution. Evaluation of complete transmigration was performed under the microscope and random fields were scanned (four fields per filter) for the presence of cells at the lower membrane side only. Invasion assays were done in a similar manner as the migration assays described above, unless the inserts were precoated with Matrigel (BD Biosciences).

Statistical methods. Continuous variables were summarized as the mean and standard deviation (SD) and the statistical comparisons between control and treatments were established by carrying out the ANOVA test or the t-test when appropriate. Dichotomous variables were summarized by absolute and/ 
A

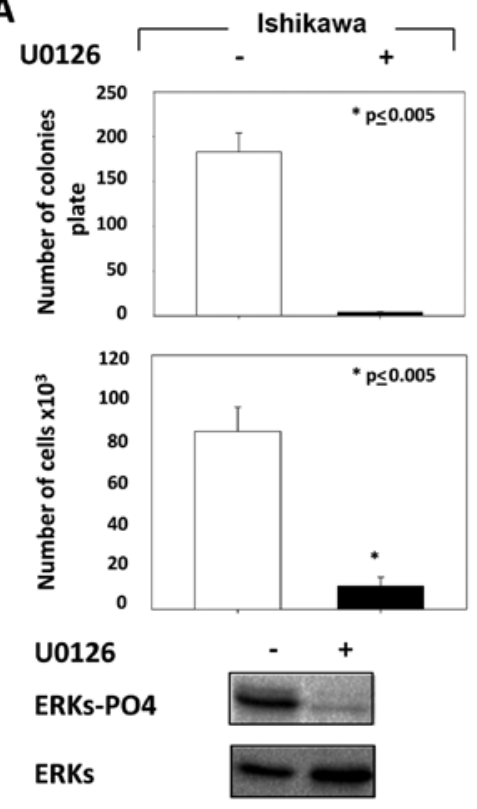

C

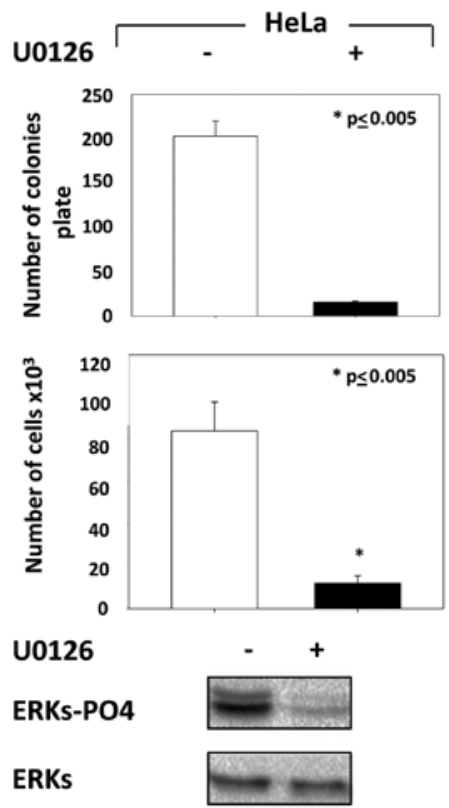

B
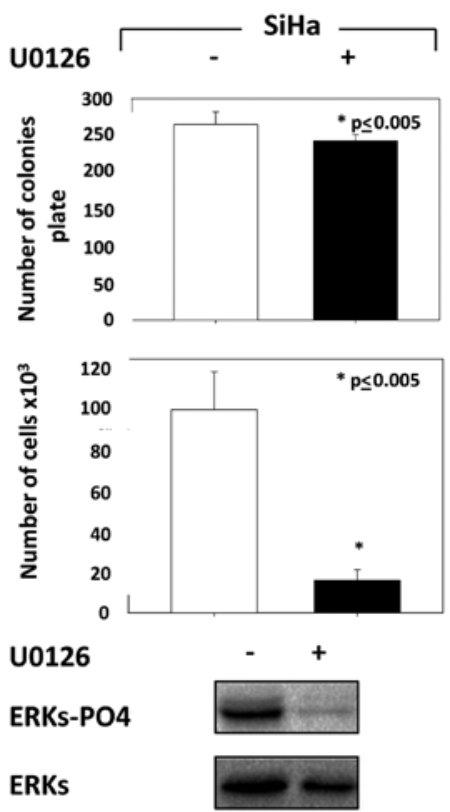

D
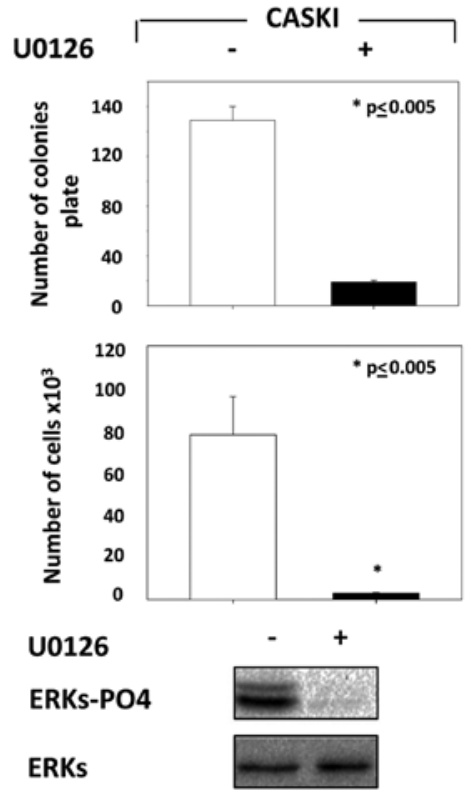

Figure 2. U0126 blocks anchorage-independent and modified monolayer growth ability of Ishikawa, SiHa, HeLa and CASKI cancer cells. (A-D, upper panel) Ishikawa, SiHa, HeLa and CASKI cells untreated (-) or treated (+) with U0126 were tested for growth in soft agar. Colonies were photographed after 14 days. (A-D, middle panel) Growth curve in modified monilayer conditions of Ishikawa, SiHa, HeLa and CASKI cells untreated (-) or treated with U0126 (+). Cells were counted after 6 days. The data shown are the mean \pm SEM of triplicates of a representative experiment. (A-D, lower panel) Expression of protein kinases ERK1/2-P04 and ERK1/2 in untreated (-) or 3 U0126-treated (+) Ishikawa, SiHa, HeLa and CASKI cancer cell lines growth in modified monolayer conditions.

or relative frequencies and statistical comparisons between control and treated groups were established by carrying out the Fisher's exact test. For multiple comparisons the level of significance was corrected according to Bonferroni correction. All tests were two-sided and were determined by Monte Carlo significance. $\mathrm{P}<0.05$ was considered statistically significant.

\section{Results}

Persistent ERK inhibition induces growtharrest in $G_{l}$, inhibition of migration/invasion and promotes anchorage-independent growth. In order to verify the effects of MEK-inhibitor, U0126, a time course experiment with or without U0126 treatment $(10 \mu \mathrm{M})$ was performed. Cells were treated with U0126 either for $3 \mathrm{~h}$ or for 4 days and subsequently processed for immunoblotting, cell count and FACS analysis. As shown in Fig. 1A and B, U0126 induced a rapid ( $3 \mathrm{~h}$ ) and persistent (4 days) decrease in phospho-active ERKs (Fig. 1A) concomitant with a decrease in the proliferation rate ranging from 65.5 to $74.9 \%$ (Fig. 1B). As shown in Fig. 1C, treatment with U0126 resulted in preferential accumulation of tumor cells in the $G_{1}-S$ phase of cell cycle, with the percentage of Ishikawa, SiHa, Caski and 
Ishikawa

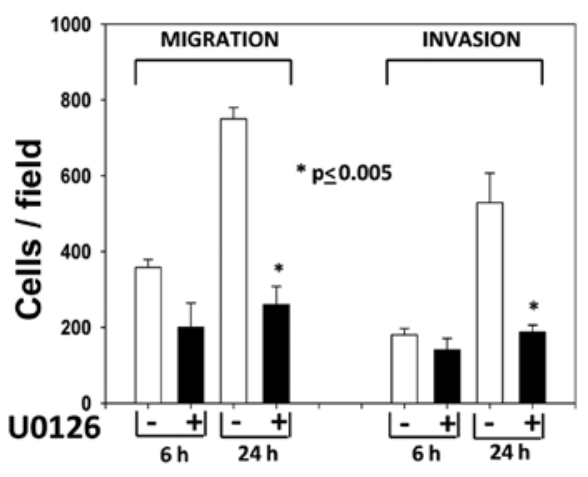

HeLa

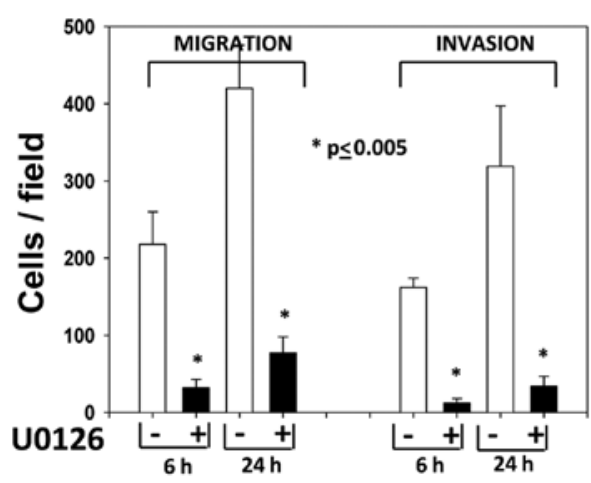

$\mathrm{SiHa}$

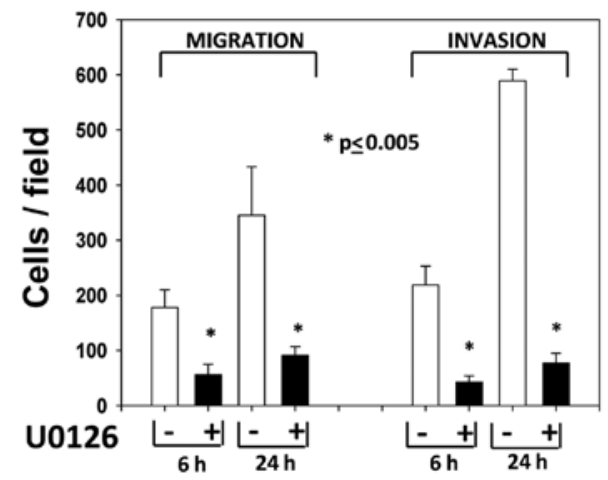

CASKI

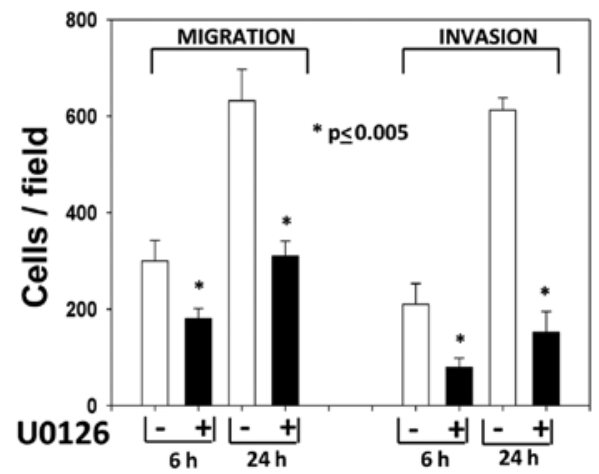

Figure 3. U0126 counteracts migration and invasion ability of Ishikawa, SiHa, HeLa and CASKI cancer cells. Transwell invasion or migration assay of Ishikawa, SiHa, HeLa and CASKI cells untreated (-) or treated (+) with U0126 for 6 or 24 h. Cells per field were counted. Similar results were obtained in three experiments.

HeLa cells in thiS phase of $61,69,74$ and $77 \%$, respectively. This phenomenon was consistent with cyclin D1 and CDK2 downregulation and p27 $7_{\text {Cip/Kip }}$ upregulation (Fig. 1D). c-Myc, one of the most tumorigenic transcription factors and downstream target of ERKs, while increasing in the expression levels comparing $3 \mathrm{~h}$ and 4 days untreated cultured cells, was early ( $3 \mathrm{~h}$ ) and persistently ( $\leq 4$ days) inhibited by the U0126 treatment as shown in Fig. 1D.

MEK/ERK inhibition is followed by downregulation of growth signal and tumorigenic molecules. To verify whether this inhibition affects anchorage-independent growth of cancer cells, we performed soft agar and cell growth assay in modified layer. Soft agar assay showed that tumor cells cultured with vehicle only formed cell aggregates of different sizes depending on the cell line used (Fig. 2A-D, upper panel). U0126 (10 $\mu \mathrm{M})$ greatly affected the propensity of tumor cells to grow in modified layer (polyHEMA coated dishes) compared to controls (Fig. 2A-D, middle panel) with a decrease in the proliferation rate of 86, 84, 93 and $89 \%$ in Ishikawa, SiHa, Caski and HeLa cell lines, respectively (ANOVA test; $\mathrm{P}<0.01$ ). The decrease of phospho-active-ERK levels in tumor cells cultured on polyHEMA-coated dishes was still present after U0126 treatments (Fig. 2A-D, lower panel). Finally, U0126 (10 $\mu \mathrm{M})$ significantly inhibited Ishikawa, SiHa, Caski and HeLa invasion and migration both at $3 \mathrm{~h}$ and 4 days after treatment, with the most evident effect at the longest time (Fig. 3). All together, these results indicate that U0126 induces growth arrest by blocking the molecular mechanism responsible of $G_{1} / S$ cell cycle phase progression and reduces the signals enabling tumorigenic and metastatic potential of tumor cells.

U0126 increases the radiosensitivity of gynecological cancer cell lines by delaying DNA repair machinery and enhancing apoptotic signaling. Tumor cells were treated with U0126 $(10 \mu \mathrm{M}) 24 \mathrm{~h}$ before the delivery of increasing doses of ionizing radiation (0-600 cGy) (Fig. 4). All cell lines were basically radioresistant at all doses tested (Fig. 4) and U0126 treatments increased the radiosensitivity with effects already evident at lowest radiation doses (Fig. 4). We further investigated whether suppressing ERKs function would influence the repair machinery of DNA double-strand breaks (DSBs) induced by irradiation. Tumor cells treated with U0126 were irradiated and the number of DNA foci representing the amount of unrepaired DSBs was counted in 4 different cell lines: i) untreated, ii) U0126-treated, iii) U0126-pretreated and iv) treated with RT (Fig. 5A-D, upper panel). For these experiments, a single dose of 4-Gy radiation was used. We have found that this dose is the most suitable to effectively distinguish the individual from combined treatments. Radiation-induced $\gamma-\mathrm{H} 2 \mathrm{AX}$ foci were readily detectable at $1 \mathrm{~h}$ of irradiation in all treated cells (Fig. 5A-D, upper panel). Interestingly, adding U0126 together with radiation led to a substantially increased number of cells retaining these foci 

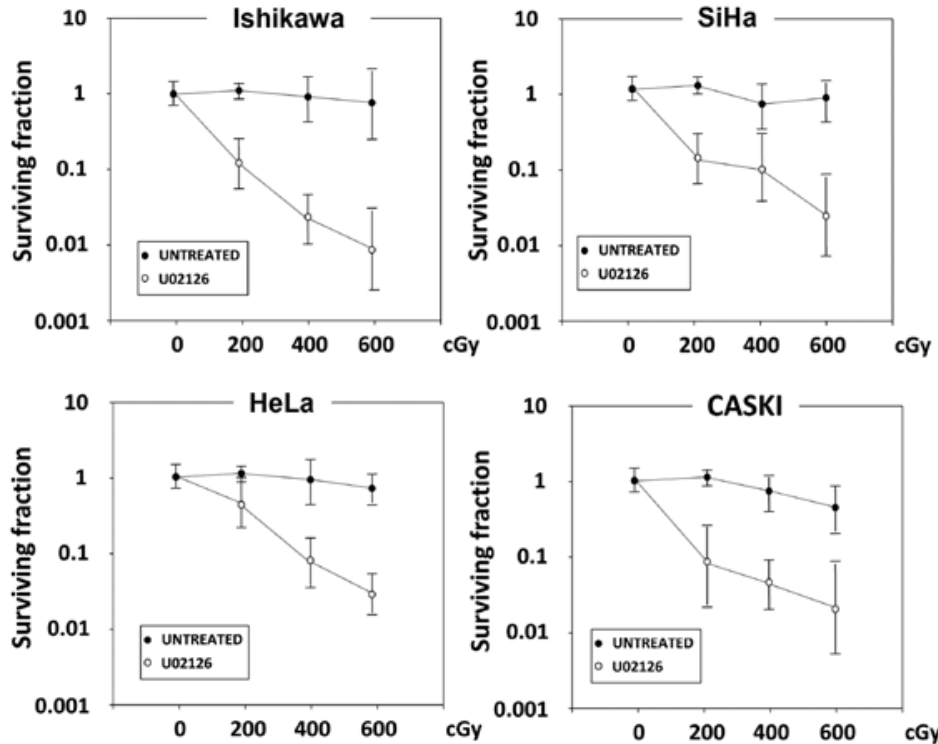

Figure 4. MEK/ERK inhibition by U0126, radiosensitizes Ishikawa, SiHa, HeLa and CASKI cells. Ishikawa, SiHa, HeLa and CASKI in the exponential phase of growth were exposed to the indicated doses of $\gamma$-radiation. Clonogenic survival was determined by counting the number of colonies containing $>50$ cells after 2 weeks of growth. The surviving fraction is shown in a semilogarithmic plot against radiation dose. Points, means from triplicate flasks from two to three independent experiments; bars, SE.

A

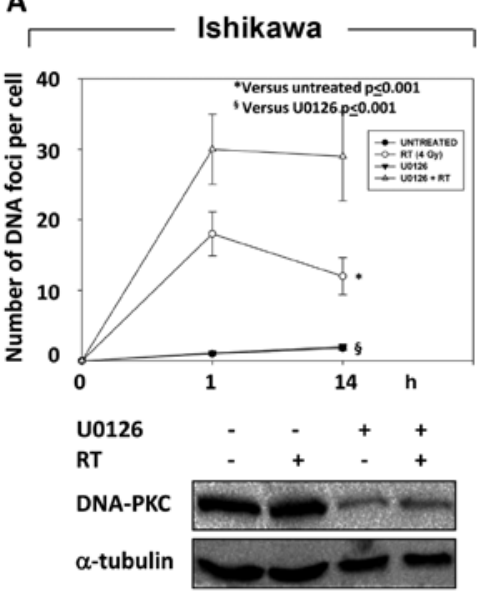

C
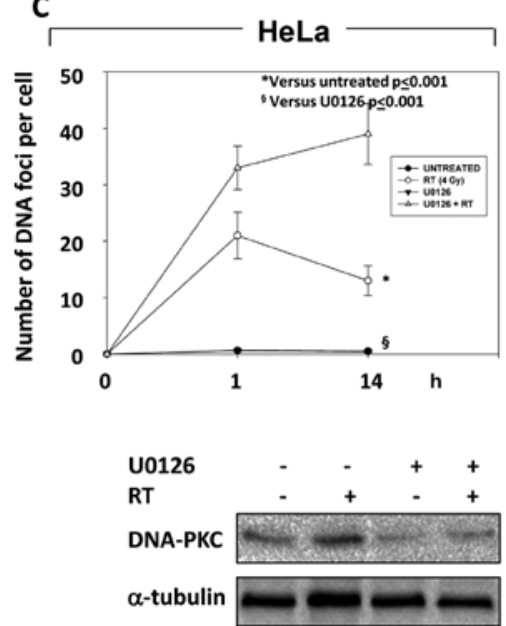

B

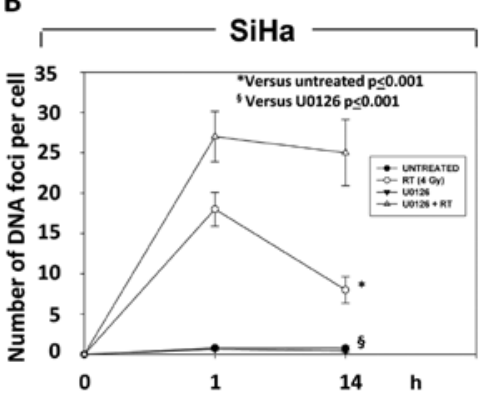

U0126

RT

DNA-PKC

$\alpha$-tubulin

D

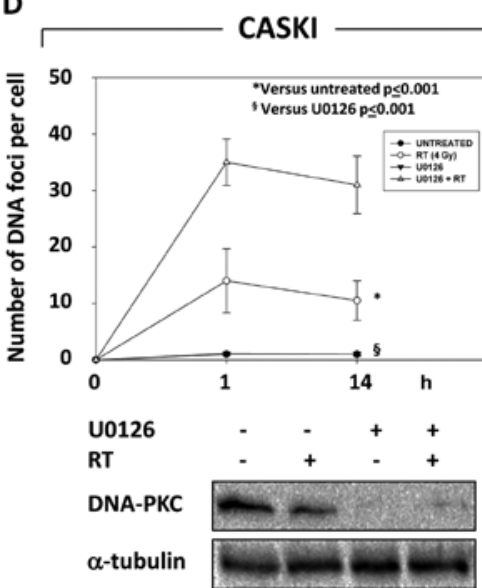

Figure 5. U0126 induces DNA damage correlated to DNA-PK protein downregulation. (A-D, upper panel) Quantification of $\gamma$-H2AX foci: DNA double-strand break profiles of Ishikawa, SiHa, HeLa and CASKI cancer cells after 24-h treatment with U0126 followed by $24 \mathrm{~h}$ of radiation treatment, as measured and categorized after $\gamma-\mathrm{H} 2 \mathrm{AX}$ immunostaining. For each category and for each dose the mean \pm SD of three experiments is shown. (A-D, lower panel) Expression of DNA-PK in Ishikawa, SiHa, HeLa and CASKI cancer cell lines pre-treated with U0126 and then exposed to 400 cGy of $\gamma$-radiation. $\alpha$-tubulin was blotted as loading control. Similar results were obtained in three experiments. 
A
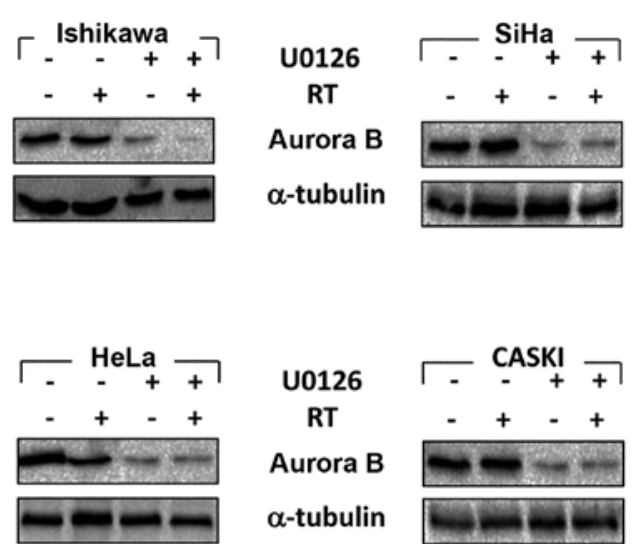

U0126

RT

Aurora B

$\alpha$-tubulin

$-+-+$

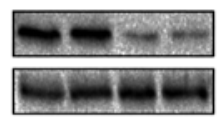

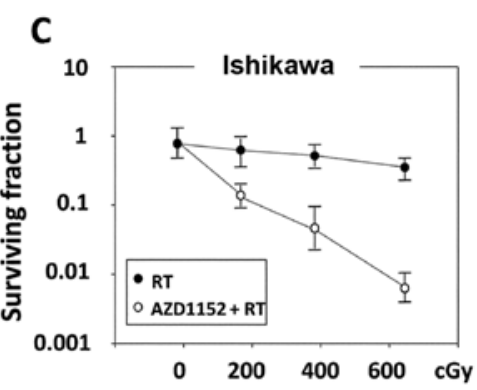

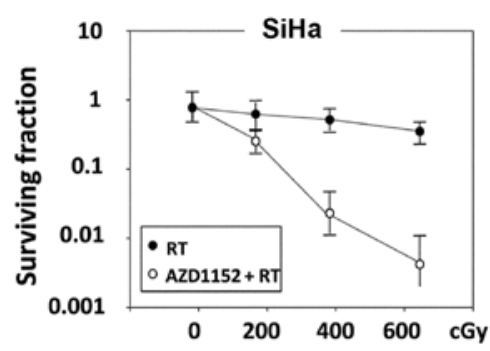

B

AZD1152

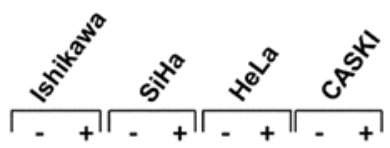

p-H3

$\alpha$-tubulin
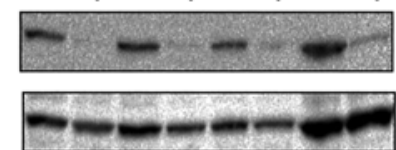

D

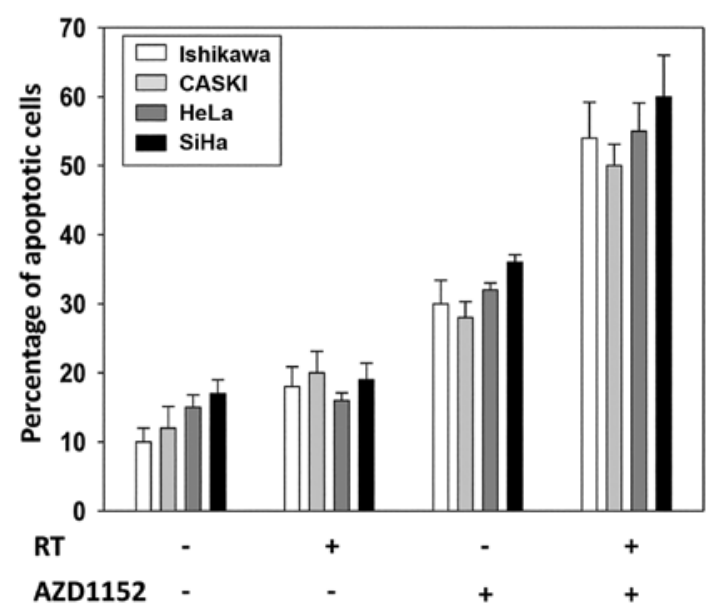

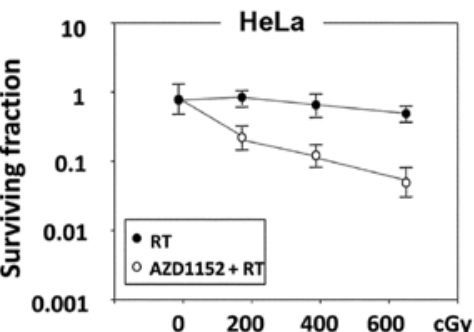

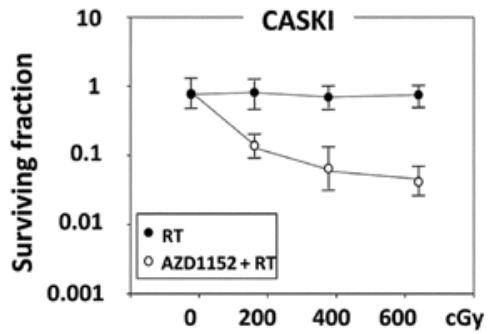

Figure 6. U0126 induces Aurora-B downregulation, inhibition of which by AZD1152 affects radiosensitivity of Ishikawa, SiHa, HeLa and CASKI cells. (A) Quantification of Aurora-B protein expression in Ishikawa, SiHa, HeLa and CASKI cancer cells lines pre-treated with U0126 and then exposed to $400 \mathrm{cGy}$ of $\gamma$-radiation. $\alpha$-tubulin was blotted as loading control. (B) Ishikawa, SiHa, HeLa and CASKI cancer cells after 24-h treatment with the Aurora-B inhibitor, AZD1152. The Aurora-B inhibition was assessed by quantifying the phosphorylation levels of H3. $\alpha$-tubulin was blotted as loading control. (C) Ishikawa, CASKI, HeLa and SiHa in the exponential phase of growth were exposed to the indicated doses of $\gamma$-radiation in the presence or absence of AZD1152. Clonogenic survival was determined by counting the number of colonies containing $>50$ cells after 2 weeks of growth. The surviving fraction is shown in a semilogarithmic plot against radiation dose. Points, means from triplicate flasks from two to three independent experiments; bars, SE. (D) Annexin V staining by FACS analysis of Ishikawa, CASKI, HeLa and $\mathrm{SiHa}$ in the exponential phase of growth were exposed to the indicated doses of $\gamma$-radiation in the presence or absence of AZD1152. Similar results were obtained in three experiments.

for $\geq 14 \mathrm{~h}$ with respect to cells treated with RT alone. The difference in the percentage of cells retaining DNA foci between the two groups was significant $(\mathrm{P}<0.01)$. Due to the fact that DNA-PKcs is usually activated in malignant tumor cells and its inactivation impairs DNA repair following ionizing irradiation we examined whether U0126 would affect DNA-PKcs expression. Of note, DNA-PKcs expression measured by western blotting at $24 \mathrm{~h}$ after treatments significantly decreased in all cell lines tested in the present study (Fig. 5A-D, lower panel).
Functional correlation between MEKs/ERKs and Aurora-B supports tumorigenic and radioresistant phenotype. We tested if the U0126-mediated effect on radiosesitization involved Aurora-B, known to play a central role in the control of DNA repair machinery. To this purpose cancer cells were cultured with U0126 $24 \mathrm{~h}$ before the delivery of ionizing radiation (4 Gy) (Fig. 6) and expression levels of Aurora-B kinases was evaluated in these cell lines. As shown in Fig. 6A, MEK/ERK inhibition reduced Aurora-B expression in all cell lines used (Fig. 6A). To verify whether in these cell lines the Aurora-B 
A

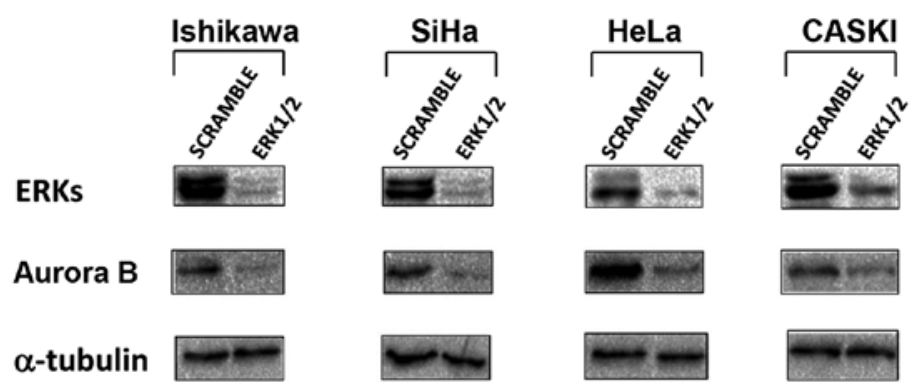

B

GROWTH ASSAY IN

GROWTH ASSAY IN CELL CULTURE MEDIUM MODIFIED MONOLAYER
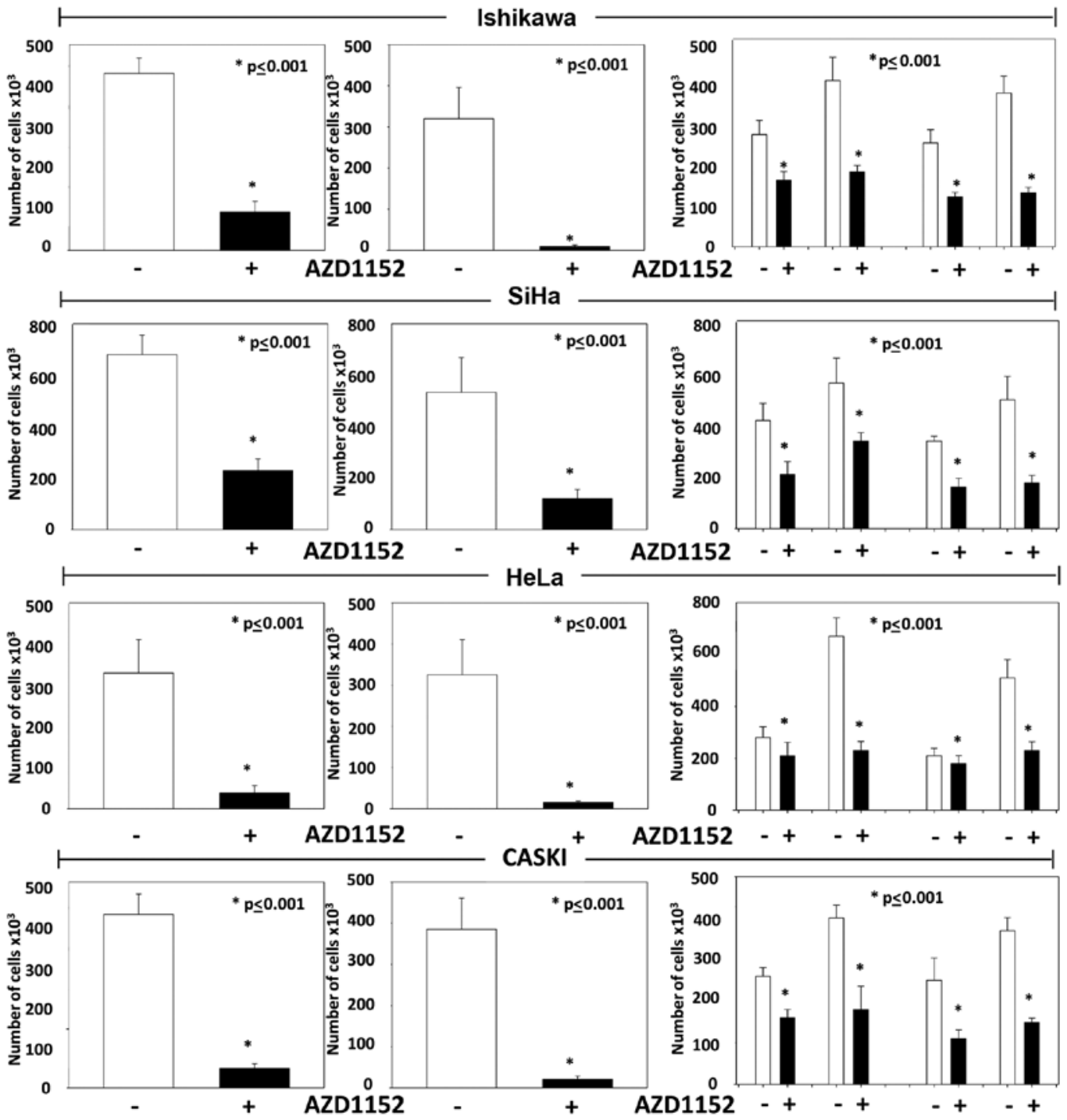

Figure 7. MEK/ERK/Aurora-B axis controls the neoplastic phenotype and radiosensitivity of gynecological cancer cells. (A) Cells were transfected with control (scramble) or ERK1/2 siRNAs and cultured for 3 days. Immunoblotting of total lysates were performed using specific antibodies recognizing the indicated proteins. $\alpha$-tubulin was blotted as loading control. (B) Cells were treated for 24 h with AZD1152 (60 nM). (B) Ishikawa, SiHa, HeLa and CASKI cells untreated (-) or treated (+) with AZD1152 were tested for growth in cells culture medium (left column) and in modified monolayer conditions (middle column). Cells were counted after 6 days (right colunm). Transwell invasion or migration assay of Ishikawa, SiHa, HeLa and CASKI cells untreated (-) or treated (+) with AZD1152 for 6 or 24 h. Cells per field were counted. Similar results were obtained in three experiments.

played a role of increased radioresponse, experiments with AZD1152, a selective Aurora-B inhibitor, were performed. The functional inhibition of Aurora-B was assessed by quantifying
p-H3, the active phosphorylated form of histone $\mathrm{H} 3$ required for normal chromosomal condensation. As shown in Fig. 6B, $24 \mathrm{~h}$ after AZD1152 $(60 \mathrm{nM})$ treatment a dramatic decrease 
in $\mathrm{p}-\mathrm{H} 3$ expression, consistent with inhibition of Aurora-B $\mathrm{H} 3$-phosphorylating activity, was observed in all cell lines. The functional inactivation of Aurora-B by AZD1152 increased the radiosensitivity of all cellular models (Fig. 6C) with a kinetic of radiosensitization similar to that observed during U0126 treatment (compare Fig. 4, with Fig. 6C). The radiosensitizing effect of AZD1152 was partially due to increased apoptotic stimulus that was greatly potentiated in association with radiotherapy (Fig. 6D) as measured by Annexin V assay. This evidence suggests a possible role of Aurora-B in the induction of pro-apoptotic stimulus upon radiation treatment.

In order to verify the existence of a direct functional link between ERK1/2 and Aurora-B, ERK1/2 RNA interference experiments were performed. Upon $72 \mathrm{~h}$ of treatment with ERK1/ERK2 siRNA, downregulation of total ERKs and Aurora-B protein levels was observed (Fig. 7A). Since MEK/ ERK silencing caused downregulation of Aurora-B expression we further studied AZD1152 effects in cellular growth, growth in modified monolayer and in migration/invasion assays. The Aurora-B inactivation by AZ1152 significantly decreased growth rate and growth in modified monolayer (Fig. 7B, left and middle panels) as well as migration/invasion (Fig. 7B, right panels) potential of all tumor cell models. This body of evidence suggests that Aurora-B mediates tumorigenic potential of gynecological cancer cell lines controlling their invasion and metastatic potential.

\section{Discussion}

Ras/MEK/ERK signaling inhibition is considered a potential novel strategic approach for therapeutic use (10). In this study, we addressed the issue of whether MEK/ERK inhibition, by interfering with Aurora-B kinase, affects the tumorigenic phenotype and radiation response of a panel of gynecological cancer cell lines. The decrease in tumorigenic signaling induced by the MEK inhibitor, U0126, clearly demonstrated that the MEK/ERK pathway is a pre-requisite for the aberrant growth in in vitro and in vivo system of these cells. Indeed, the permanent phospho-active ERK inhibition in all tumor cell lines used was followed by growth arrest, loss of both anchorage-independent growth and migration/invasion potential. Importantly, MEK/ERK inhibition had significant effects on cell cycle distribution causing an increased accumulation of cells in $\mathrm{G}_{1}$ phase, ranging from $65-80 \%$ depending on cell line in 24-h treatment. This anti-growth response is countersigned by a decreased levels of a number of proteins that are globally correlated with cell cycle progression (CDK2, Myc and cyclin D1) or with cell cycle inhibition (p27).

Moreover, the effects of Ras/MEKs/ERKs inhibition and the downstream target pathways on radiation response have not yet been studied in detail in gynecological tumors. With respect to intrinsic cellular radiation sensitivity, it is known that cells are more responsive to the ionizing radiation effects in $\mathrm{G}_{2} / \mathrm{M}$ than in $G_{1}$ phase. In these lines the MEK/ERK inhibitor led a significant percent of cells $(65-80 \%)$ into $G_{1}$ phase of the cell cycle within 24-h treatment, while radiation, combined with the MEK inhibitor, in just one hour gained the main effects on DNA foci. From this we can conclude that MEK inhibitor does not lower RT-mediated DNA lesions but rather helps radiosensitization as we previously reported in RMS in vivo and in vitro models $(32,34)$. Effects of combined MEK/ERK inhibitor and radiation are particularly evident in the persistence of DNA foci, which decays within $14 \mathrm{~h}$ after radiation alone. Our data point to a possible loss of DNA repair mechanisms, resulting in MEK/ERK inhibitor-induced DNA-PKcs downregulation. Our study for the first time, demonstrated a close correlation between MEK/ERK and Aurora-B in a functional axis where the loss of ERK causes Aurora-B downregulation enhancing the response to DNA damage by radiation. The idea that MEK/ ERK is a therapeutical target which enhances radiosensitivity was corroborated by the MEK inhibitor-mediated loss of DNA-PKcs, a member of the DNA repair machinery.

The close correlation between MEK/ERK signaling pathway and Aurora-B is of particular significance in light of recent findings reporting that MAPKs and Aurora-B cooperate in promoting invasive growth (41-45), tumorigenic potential $(46,47)$ and radioresistance phenomena (42-44). We successfully demonstrated that MEK/ERK kinases regulate Aurora-B expression, suggesting that MAPK pathway and Aurora-B kinase rely on a functional collaboration, which is most likely required to sustain the tumorigenic and radioresistant phenotype of the gynecological cancer cells. This evidence was supported by the findings that selective Aurora-B inhibition by AZD1152 recovered radioprotection, induced apoptotic events and elicited anti-growth responses, similarly to that seen after MEK/ERK inhibition. Aurora-B expression dependence on active ERKs is also demonstrated by the siRNA-mediated ERK silencing, which induced downregulation of Aurora-B protein expression. Collectively, our findings suggest that the modulation of MEK/ERK signaling pathway has a strong impact on Aurora-B kinase resulting in reduced tumorigenic radioresistant phenotype in all gynecological cancer cell lines.

In conclusion, results from our studies on MEK/ERK/ Aurora-B axis inhibition by MEK/ERK inihibitor can be considered of high relevance in molding the new therapeutic approaches in treating tumorigenesis such as endometrial, cervical and vaginal cancers.

\section{References}

1. Weiderpass $\mathrm{E}$ and Labrèche $\mathrm{F}$ : Malignant tumors of the female reproductive system. Saf Health Work 3: 166-180, 2012.

2. Sharma DN: Radiation in vulvar cancer. Curr Opin Obstet Gynecol 24: 24-30, 2012.

3. Wright JD, Barrena Medel NI, Sehouli J, Fujiwara K and Herzog TJ: Contemporary management of endometrial cancer. Lancet 379: 1352-1360, 2012.

4. Willmott LJ and Monk BJ: Cervical cancer therapy: current, future and anti-angiogensis targeted treatment. Expert Rev Anticancer Ther 9: 895-903, 2009.

5. McKenna WG, Weiss MC, Bakanauskas VJ, et al: The role of the H-ras oncogene in radiation resistance and metastasis. Int $\mathrm{J}$ Radiat Oncol Biol Phys 18: 849-859, 1990.

6. Jones HA, Hahn SM, Bernhard E and McKenna WG: Ras inhibitors and radiation therapy. Semin Radiat Oncol 11: 328-337, 2001.

7. Dar AA, Goff LW, Majid S, Berlin J and El-Rifai W: Aurora kinase inhibitors-rising stars in cancer therapeutics? Mol Cancer Ther 9: 268-278, 2010.

8. O'Neill E and Kolch W: Conferring specificity on the ubiquitous Raf/MEK signaling pathway. Br J Cancer 90: 283-288, 2004.

9. Murphy LO and Blenis J: MAPK signal specificity: the right place at the right time. Trends Biochem Sci 31: 268-275, 2006.

10. Kohno M and Pouyssegur J: Pharmacological inhibitors of the ERK signaling pathway: application as anticancer drugs. Prog Cell Cycle Res 5: 219-224, 2003. 
11. Faivre S, Djelloul S and Raymond E: New paradigms in anticancer therapy: targeting multiple signaling pathways with kinase inhibitors. Semin Oncol 33: 407-420, 2006.

12. Liu D, Liu Z, Condouris S and Xing M: BRAF V600E maintains proliferation, transformation, and tumorigenicity of BRAFmutant papillary thyroid cancer cells. J Clin Endocrinol Metab 92: 2264-2271, 2007.

13. Martinelli S, McDowell HP, Vigne SD, Kokai G, Uccini S and Tartaglia M: RAS signaling dysregulation in human embryonal rhabdomyosarcoma. Genes Chromosomes Cancer 48: 975-982, 2009.

14. Marshall CJ: The ras oncogenes. J Cell Sci 10: 157-169, 1998.

15. Gupta AK, Bakanauskas VJ, Cerniglia GJ, et al: The Ras radiation resistance pathway. Cancer Res 61: 4278-4282, 2001.

16. Toulany M, Baumann $M$ and Rodemann HP: Stimulated PI3K-AKT signaling mediated through ligand or radiationinduced EGFR depends indirectly, but not directly, on constitutive K-Ras activity. Mol Cancer Res 5: 863-872, 2007.

17. Adhikary $\mathrm{S}$ and Eilers M: Transcriptional regulation and transformation by Myc proteins. Nat Rev Mol Cell Biol 6: 635-645, 2005.

18. Mammas IN, Zafiropoulos A and Spandidos DA: Involvement of the ras genes in female genital tract cancer. Int J Oncol 26 : $1241-1255,2005$.

19. Carmena M and Earnshaw WC: The cellular geography of aurora kinases. Nat Rev Mol Cell Biol 4: 842-854, 2003.

20. Ducat D and Zheng Y: Aurora kinases in spindle assembly and chromosome segregation. Exp Cell Res 301: 60-67, 2004.

21. Marumoto T, Zhang D and Saya H: Aurora A, a guardian of poles. Nat Rev Cancer 5: 42-50, 2005

22. Fu J, Bian M, Jiang Q and Zhang C: Roles of Aurora kinases in mitosis and tumorigenesis. Mol Cancer Res 5: 1-10, 2007.

23. Kobayashi M, Nakamura S, Ono T, et al: Analysis of aurora kinase expressions and cell cycle regulation by Aurora $\mathrm{C}$ in leukemia cells. Blood (ASH Annual Meeting Abstracts) 108: $1366,2006$.

24. Slattery SD, Mancini MA, Brinkley BR and Hall RM: Aurora-C kinase supports mitotic progression in the absence of Aurora-B. Cell Cycle 8: 2986-2997, 2009.

25. Zhang X: Aurora kinases. Curr Biol 18: R146-R148, 2008.

26. Carvajal RD, Tse A and Schwartz GK: Aurora kinases: new targets for cancer therapy. Clin Cancer Res 12: 6869-6875, 2006.

27. Girdler F, Gascoigne KE, Eyers PA, et al: Validating Aurora B as an anti-cancer drug target. J Cell Sci 119: 3664-3675, 2006

28. Smith SL, Bowers NL, Betticher DC, et al: Overexpression of Aurora B kinase (AURBK) in primary non-small cell lung carcinoma is frequent, generally driven from one allele, and correlates with the level of genetic instability. Br J Cancer 93 : 719-729, 2005.

29. Gautschi O, Heighway J, Mack PC, Purnell PR, Lara PN Jr and Gandara DR: Aurora kinases as anticancer drug targets. Clin Cancer Res 14: 1639-1648, 2008.

30. Keen M and Taylor S: Mitotic drivers - inhibitors of the Aurora B kinase. Cancer Metastasis Rev 28: 185-195, 2009.

31. Bonet C, Giuliano S, Ohanna M, et al: Aurora B is regulated by the mitogen-activated protein kinase/extracellular signalregulated kinase (MAPK/ERK) signaling pathway and is a valuable potential target in melanoma cells. J Biol Chem 287: 29887-29898, 2012
32. Marampon F, Gravina GL, Di Rocco A, et al: MEK/ERK inhibitor U0126 increases the radiosensitivity of rhabdomyosarcoma cells in vitro and in vivo by downregulating growth and DNA repair signals. Mol Cancer Ther 10: 159-168, 2011

33. Marampon F, Bossi G, Ciccarelli C, et al: MEK/ERK inhibitor U0126 affects in vitro and in vivo growth of embryonal rhabdomyosarcoma. Mol Cancer Ther 8: 543-551, 2009.

34. Marampon F, Ciccarelli C and Zani BM: Down-regulation of c-Myc following MEK/ERK inhibition halts the expression of malignant phenotype in rhabdomyosarcoma and in non musclederived human tumors. Mol Cancer 5: 31, 2009.

35. Allalunis-Turner MJ, Zia PK, Barron GM, Mirzayans R and Day RS III: Radiation-induced DNA damage and repair in cells of a radiosensitive human malignant glioma cell line. Radiat Res 144: 288-293, 1995.

36. Lees-Miller SP, Godbout R, Chan DW, Weinfeld M, Day RS III, Barron GM and Allalunis-Turner J: Absence of p350 subunit of DNA-activated protein kinase from a radiosensitive human cell line. Science 267: 1183-1185, 1995.

37. Tian X, Chen G, Xing H, Weng D, Guo Y and Ma D: The relationship between the down-regulation of DNA-PKcs or Ku70 and the chemosensitization in human cervical carcinoma cell line HeLa. Oncol Rep 18: 927-932, 2007.

38. Gerweck LE, Vijayappa S, Kurimasa A, Ogawa K and Chen DJ: Tumor cell radiosensitivity is a major determinant of tumor response to radiation. Cancer Res 66: 8352-8355, 2006.

39. van Gent DC, Hoeijmakers JH and Kanaar R: Chromosomal stability and the DNA double-stranded break connection. Nat Rev Genet 2: 196-206, 2001.

40. Lowry OH, Rosebrough NJ, Farr AL and Randall RJ: Protein measurement with the Folin phenol reagent. Biol Chem 193: 265-275, 1951.

41. Mori S, Chang JT, Andrechek ER, et al: Anchorage-independent cell growth signature identifies tumors with metastatic potential. Oncogene 28: 2796-2805, 2009.

42. Sak A, Stuschke M, Groneberg M, Kübler D, Pöttgen C and Eberhardt WE: Inhibiting the Aurora B kinase potently suppresses repopulation during fractionated irradiation of human lung cancer cell lines. Int J Radiat Oncol Biol Phys 84: 492-499, 2012.

43. Borges KS, Castro-Gamero AM, Moreno DA, et al: Inhibition of Aurora kinases enhances chemosensitivity to temozolomide and causes radiosensitization in glioblastoma cells. J Cancer Res Clin Oncol 138: 405-414, 2012

44. Niermann KJ, Moretti L, Giacalone NJ, et al: Enhanced radiosensitivity of androgen-resistant prostate cancer: AZD1152mediated Aurora kinase B inhibition. Radiat Res 175: 444-451, 2011.

45. Kosik A, Bekier ME, Katusin JD, et al: Investigating the role of Aurora kinases in RAS signaling. J Cell Biochem 106: 33-41, 2009.

46. Ma Y, Weimer J, Fredrik R, et al: Aurora kinase inhibitor AZD1152 has an additional effect of platinum on a sequential application at the human ovarian cancer cell line SKOV3. Arch Gynecol Obstet 288: 173-182, 2013.

47. Baldini E, Sorrenti S, D'Armiento E, et al: Aurora kinases: new molecular targets in thyroid cancer therapy. Clin Ter 163: e457-462, 2012. 